\title{
Laboratory tools and e-learning elements in training of acousto-optics
}

\section{Attila Barócsi, Sándor Lenk, Ferenc Ujhelyi, Tamás Majoros, Paál Maák}

Attila Barócsi, Sándor Lenk, Ferenc Ujhelyi, Tamás Majoros, Paál Maák, "Laboratory tools and e-learning elements in training of acousto-optics," Proc. SPIE 9793, Education and Training in Optics and Photonics: ETOP 2015, 97930U (8 October 2015); doi: 10.1117/12.2223088

SPIE Event: Education and Training in Optics and Photonics: ETOP 2015, 2015, Bordeaux, France 


\title{
Laboratory tools and e-learning elements in training of acousto-optics Attila Barócsi*, Sándor Lenk, Ferenc Ujhelyi, Tamás Majoros, Paál Maák Dept. of Atomic Physics, Budapest Univ. of Technology and Economics, 8 Budafoki, Budapest, Hungary H-1111
}

\begin{abstract}
Due to the acousto-optic (AO) effect, the refractive index of an optical interaction medium is perturbed by an acoustic wave induced in the medium that builds up a phase grating that will diffract the incident light beam if the condition of constructive interference is satisfied. All parameters, such as magnitude, period or phase of the grating can be controlled that allows the construction of useful devices (modulators, switches, one or multi-dimensional deflectors, spectrum analyzers, tunable filters, frequency shifters, etc.) The research and training of acousto-optics have a long-term tradition at our department. In this presentation, we introduce the related laboratory exercises fitted into an e-learning frame. The BSc level exercise utilizes a laser source and an AO cell to demonstrate the effect and principal AO functions explaining signal processing terms such as amplitude or frequency modulation, modulation depth and Fourier transformation ending up in building a free space sound transmitting and demodulation system. The setup for MSc level utilizes an AO filter with mono- and polychromatic light sources to learn about spectral analysis and synthesis. Smart phones can be used to generate signal inputs or outputs for both setups as well as to help students’ preparation and reporting.
\end{abstract}

Keywords: acousto-optics, tunable filter, signal processing, spectral analysis, smart phones, free space transmission, laboratory tool, e-learning elements

\section{INTRODUCTION}

Acousto-optics in the scientific literature will definitely refer to the more than 50 years of technological progress initiated by the development of the laser in 1960. It became obvious that utilizing the acousto-optic (AO) effect provides an elegant way for influencing photons by altering the refractive index of the medium in which the light propagates.

The story begun in 1922 when L. Brillouin was investigating the properties of acoustic waves induced thermally in liquids and solids ${ }^{1}$. According to his model, the acoustic fluctuations yielded a periodic change of the density of the propagation medium resulting in an alteration of the dielectric permittivity. If so, this change could be determined from the scattering pattern of a light wave with appropriately short wavelength. Brilluein himself proposed the analogy between this scattering phenomenon and the diffraction of light from periodic structures (studied by W. H. Bragg and W. L. Bragg in the 1910s). Thus, the incidence angle of the light beam must be chosen so as to obtain constructive interference. A principal difference, he argued, was that an acoustic wave created a sinusoidal grating that allowed only two $( \pm 1)$ diffraction orders and the corresponding critical angles. Later, this phenomenon was termed Bragg diffraction. There is another distinction from an optical grating: the acoustic grating propagates with the speed of sound, hence the frequency of light is Doppler shifted by the acoustic frequency with opposite signs for the two orders.

There was 10 more years passed till the experiments were realized by P. Debye and F. W. Sears, as well as R. Lucas and P. Biquard demonstrating the AO effect by ultrasonic waves in liquids and solids ${ }^{2,3}$. The results were, however, surprisingly different from Brilluein's predictions: the critical angles could not be demonstrated and, for intense acoustic field, more than two orders appeared due to the small interaction volume as Debye and Sears realized. The finding is worth emphasizing that constructive interference can be maintained in a wide angular range: this fact allows for a finite interaction bandwidth being one of the most important parameters in terms of practical application of AO devices.

Although the AO effect was nicely formulated analytically later verified by a few experiments, not until the 'laser era' its practical application advanced. Then a boom in applied acousto-optics initiated from the various light controlling AO devices, such as traveling and standing wave modulators ${ }^{4}$ and switches ${ }^{5}$, single or multi-dimensional deflectors ${ }^{6}$, tunable filters $^{7}$ and frequency shifters ${ }^{8}$ to the sophisticated AO signal processing systems such as a real time power spectrum analyzer $^{9}$, direction of arrival processor ${ }^{10}$, or 3-dimensional (3D) AO light steering in multi-photon microscopy ${ }^{11}$. This progress seemed to cease by the spreading of laser diodes allowing a direct fast modulation of light. However, it has again been boosted by the utilization of the AO effect in the dispersion control and shaping of ultrafast laser beams ${ }^{12}$.

*barocsi@eik.bme.hu; phone +36 1 463-1374; fax +36 1 463-4194; www.fat.bme.hu

Education and Training in Optics and Photonics: ETOP 2015, edited by Eric Cormier, Laurent Sarger Proc. of SPIE Vol. 9793, 97930U · (c) 2015 SPIE, IEEE, OSA, ICO · doi: 10.1117/12.2223088 
The research and development of AO devices, systems and technology as well as the accompanying training of acoustooptics at different levels of the education have a long-term tradition at the Department of Atomic Physics dating back to the 1980s. Although training starts at BSc level of higher education in physics, several demonstration activities involving acousto-optics present each year for secondary school pupils orienting them among faculties and specializations. The training continues at MSc then PhD levels offering research activities in the topic ${ }^{13}$. Next, we introduce this training set in the framework of two related laboratory exercises set at BSc and MSc levels. Recently, e-learning elements have been being developed accompanying the training to help students' laboratory preparation, work and reporting.

\section{TRAINING THE FUNDAMENTALS OF ACOUSTO-OPTICS}

Within the framework of the BSc in Physics program, basics of acousto-optics are demonstrated in the Advanced Physics Laboratory 2 simultaneously with Optics in the 4th semester, in which subject its fundamental theory is taught, as summarized below.

\subsection{Basic concepts of acousto-optics}

Due to the photoelastic effect, propagation of an acoustic wave in a medium induces a periodic change of its refractive index yielding a phase grating that travels at a speed of the acoustic wave in the medium. The gratings constant equals to the acoustic wavelength $\lambda_{a}$, whereas the magnitude of the grating is proportional to the acoustic amplitude $S_{0}$ and the magnitude $p$ of the photoelastic effect. According to Figure 1a, consider an acoustic plane wave with wave vector $\mathbf{k}_{a}=k_{a} \hat{\mathbf{x}}=\left(2 \pi / \lambda_{a}\right) \hat{\mathbf{x}}$ and angular frequency $\omega_{a}$ that propagates in the $\mathbf{x}=x \hat{\mathbf{x}}$ direction of the $x z$ plane

$$
s(x, t)=S_{0} \cos \left(\omega_{a} t-k_{a} x\right),
$$

and an optical plane wave with $\mathbf{k}_{0}$ and $\omega_{0}$ propagating in the direction characterized by the internal angle $\theta_{0}$ from the $x$ axis. Propagation of the optical beam in the medium is given by the wave equation

$$
\nabla^{2} \mathbf{E}(\mathbf{r}, t)=\frac{n^{2}(x, t)}{c^{2}} \frac{\partial^{2} \mathbf{E}(\mathbf{r}, t)}{\partial t^{2}},
$$

where $c$ is the speed of light in vacuum and $n(x, t)$ is the refractive index of the medium (Figure 1b), approximated as

$$
n(x, t)=n-\frac{1}{2} p n^{3} s(x, t)=n-\delta n \cos \left(\omega_{a} t-k_{a} x\right)
$$

with effective index $n$ for small $\delta n=p n^{3} S_{0} / 2$ magnitude of the index change. As the electromagnetic field is periodic, it can be decomposed into a Fourier-series ${ }^{14}$. Putting the Fourier decomposition into the wave equation and omitting higher order components, for scalar, slowly varying field, i.e. $d E_{m} / d z<<k_{v}\left|E_{m}(z)\right|$, yields

$$
\frac{d E_{m}}{d z}+\frac{k_{v} \delta n}{2 \cos \theta_{0}}\left(E_{m+1}-E_{m-1}\right)=-j \frac{m k_{a}}{\cos \theta_{0}}\left(\sin \theta_{0}-\sin \theta_{B}\right) E_{m}
$$

for the $E_{m}=E_{m}(z)$ amplitude of order $m=\{0, \pm 1, \pm 2, \ldots\}$ with wave number $k_{v}=2 \pi / \lambda_{v}$ in vacuum and the Bragg angle

$$
\sin \theta_{B}=\frac{k_{a}}{2 k_{0}}
$$

ensuring constructive interference. Equation (5) can be generalized by applying the conservation of momentum for the interaction in terms of the wave vector matching (Figure 1c),

$$
\mathbf{k}_{0}+m \mathbf{k}_{a}=\mathbf{k}_{m},
$$

whereas the energy is conserved through the frequency condition of

$$
\omega_{0}+m \omega_{a}=\omega_{m} .
$$




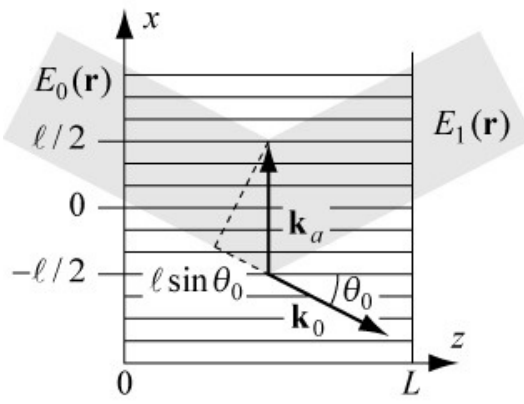

(a)

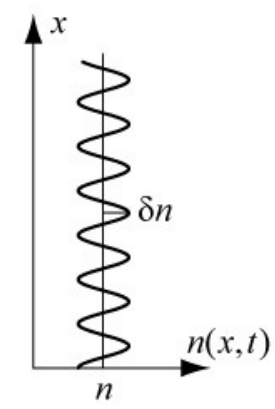

(b)

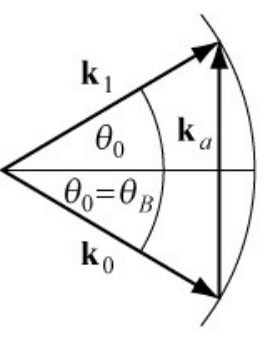

(c)

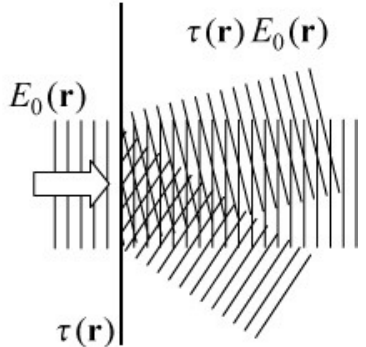

(d)

Figure 1. Geometry of the AO interaction (a) with the refractive index change (b) and the wave vector matching for $m=1$ at the Bragg condition (c). Association can also be made to the scalar diffraction model (d) that applies field decomposition into plane waves at an aperture with complex transmittance function $\tau(\mathbf{r})$.

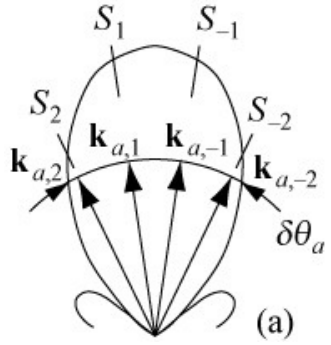

(a)

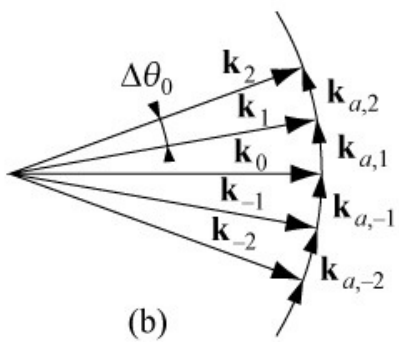

(b) (c)

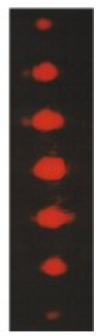

Figure 2. The effect of the acoustic divergence (a) on the appearance of higher diffraction orders (b). Demonstration of many AO diffraction orders (c).

Although the derivation of the so called coupled wave equation (4) being fundamental in the quantitative description of the AO effect goes beyond this training level, its visual interpretation in the course of the laboratory exercise is well fit into the scalar diffraction theory using Fourier decomposition as taught in the course of subject Optics (Figure 1d). This aspect also explains the term Bragg diffraction occurring from thick volume grating as opposed to a thin grating producing many diffraction orders. In general, higher orders may exist if the term in Equation (5) is small as demonstrated experimentally in Figure 2c. We recall also on the basis of the scalar diffraction theory that the full width divergence $\delta \theta_{a}$ and angular spectrum $S\left(\theta_{a}\right)$ of a rectangular sound column with length $L$ is approximated as

$$
\delta \theta_{a} \approx \frac{\lambda_{a}}{L}, \quad S\left(\theta_{a}\right)=S_{0} \operatorname{sinc} \frac{\pi \theta_{a}}{\delta \theta_{a}} .
$$

From Equation (5) and Figure 1c, we know that the diffracted orders are separated by $2 \sin \theta_{B}=\lambda_{0} / \lambda_{a}$, so the condition

$$
\delta \theta_{a} \gg \Delta \theta_{0} \approx 2 \theta_{B}=\frac{\lambda_{0}}{\lambda_{a}}=\frac{\lambda_{v}}{n \lambda_{a}}
$$

ensures the appearance of higher orders, as depicted in Figures 2a-b.

On the other hand, the theory of the AO effect is introduced in Optics in a far simpler way by integrating the elementary $d r$ complex amplitude reflectance coefficients upon planar layers separated by $d x$ distance along the $x$ direction (Figure 1a) to get the total amplitude reflectance ${ }^{15}$

$$
r=\int_{-\ell / 2}^{\ell / 2} \mathrm{e}^{\mathrm{j} 2 k_{0} x \sin \theta_{0}} \frac{d r}{d x} d x, \quad \frac{d r}{d x}=\frac{d r}{d n} \frac{d n}{d x} .
$$


The first derivative $d r / d n$ of the local change of amplitude reflectance $d r / d x$ can be obtained from the Fresnel equations whereas the second term is calculated from Equation (3). This approach explains the term Bragg reflection.

\subsection{Training setup}

Figure 3a shows the basic AO experimental setup. The optical elements are built upon a pre-aligned linear optical stage of to minimize the necessary adjustments. As seen from Figure $3 \mathrm{~b}$, the AO cell is mounted on a rotator that allows adjusting of the Bragg angle in a vertical plane. This translates to one vertical degree of freedom for adjusting the detector. A horizontal translator is also placed under the AO cell to provide fine tuning of its entrance aperture while maintaining the beam propagation in the horizontal axis of the optical stage. Key elements in the setup are a frequency generator with internal/external AM/FM modulator and a combined AO driver with switching modulator and power amplifier. The generator operates in the radio frequency (RF) range of $10 \mathrm{MHz}$ to $100 \mathrm{MHz}$ and its output is amplified up to a maximum of undistorted $1 \mathrm{~W}$. A $633 \mathrm{~nm}$ He-Ne laser with $3 \mathrm{~mW}$ output optical power is used as a light source.

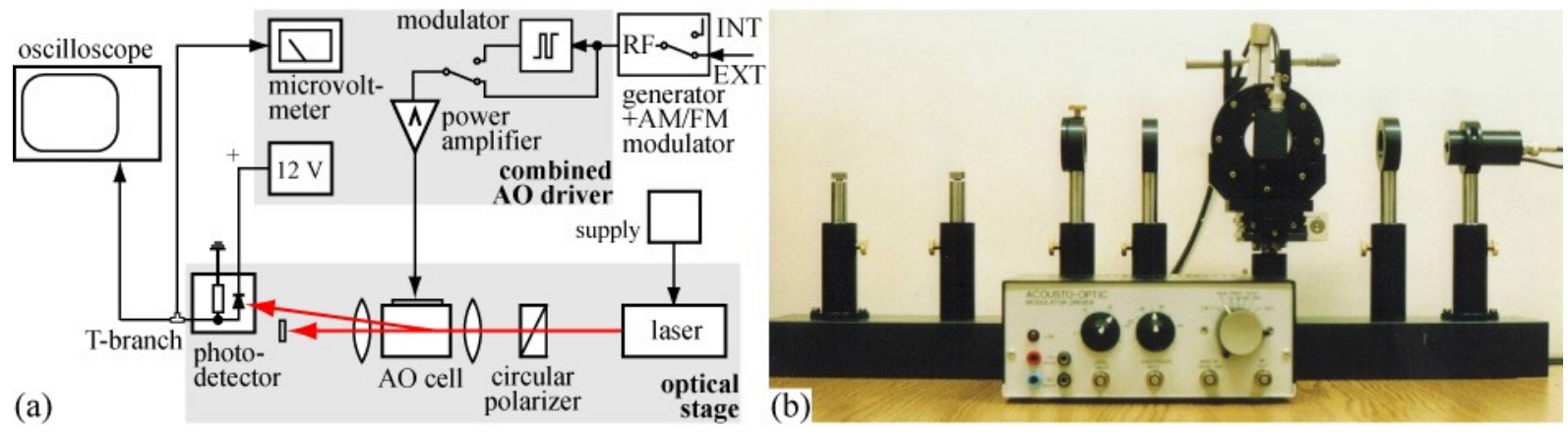

Figure 3. Block scheme (a) and optical stage (b) of the basic AO experimental setup with many in-house made components

As a basic experiment, the $\mathrm{AO}$ interaction geometry can be studied according to Figure 1a together with the visualization of the diffraction orders. Given the refractive index of the $\mathrm{AO}$ cell made of $\mathrm{TeO}_{2}$, the sound velocity $v_{a}$ can be calculated from the external refraction geometry measured at the Bragg condition of Figure 1c. Also, as a practical application of the AO cell, the light deflector concept (Figure 4a) can be introduced by measuring the deflection angle $\Theta$ defined as the total external angle between the 1st and 0th order beam. Using Snells's law

$$
\Theta=2 \arcsin \left(n \sin \theta_{0}\right)=2 \arcsin \frac{\lambda_{v}}{2 \lambda_{a}}=2 \arcsin \frac{\lambda_{v} f_{a}}{2 v_{a}} .
$$

For small angles, Equation (11) simplifies to a linear $\Theta\left(f_{a}\right)$ relationship versus acoustic frequency $f_{a}$.

Using the photodetector and the microvolt-meter or the oscilloscope, the intensities of the diffracted orders can be determined. At the Bragg condition, the $\eta_{d}$ diffraction efficiency is defined as the ratio of the intensity of the 1st order beam to that of the 0th order one being equal to the peak reflectance that can be obtained upon evaluating Equation (10):

$$
\eta_{d} \equiv \frac{I_{1}}{I_{0}}=\left|r\left(\theta_{B}\right)\right|^{2} .
$$

At low acoustic field intensities, the efficiency is proportional to the acoustic intensity $I_{a}=\rho v_{a}^{3} S_{0}^{2} / 2$ with $\rho$ being the density of the medium or, more conveniently, to the acoustic power $P_{a}=I_{a} L H$ with $L$ and $H$ being the interaction length defined in Figure 1a and the width of the sound column, respectively. Idealizing that the acoustic power equals to the input electric power, $P_{a} \approx P_{i n}$, the linear $\eta_{d}\left(P_{a}\right)$ relationship can be measured. To do so, the output level of the RF generator is adjustable by a rotary switch with pre-calibrated power levels. 


\section{ADVANCED TRAINING OF ACOUSTO-OPTICS}

As the RF drive power of the AO cell increases, the linear $\eta_{d}\left(P_{a}\right)$ relationship will not hold. A more precise analysis (in which depletion of the incident beam is also considered) reveals the following expression:

$$
\eta_{d}\left(P_{a}\right)=\sin ^{2}\left(C \sqrt{P_{a}}\right),
$$

where all material and geometric parameters are embedded in constant $C$. This diffraction efficiency function can also be measured versus RF driving power in the basic laboratory and will depict a curve similar to that of Figure 6a. Going to advance level, Equation (13) can be regarded as the modulation transfer function of the AO cell when considered as a light intensity modulator. To measure the temporal response of the AO cell upon an input signal, the basics of signal modulation must be introduced. Amplitude modulation (AM) of an input electric signal is the simplest mode that can be realized by means of an $\mathrm{AO}$ modulator (Figure $4 \mathrm{~b}$ ).
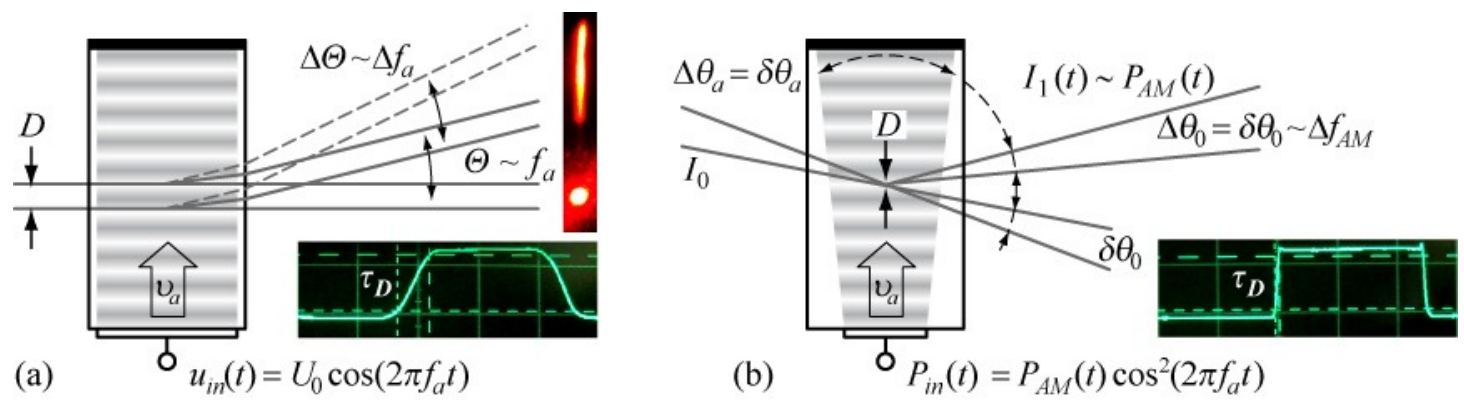

Figure 4. The AO cell operated as a light deflector (a) and light modulator (b) with characteristic input and output signals. Insets of (a) show the $\Delta \Theta$ scanning range and the $\tau_{D}$ transit time of the deflector. Inset of (b) shows $\tau_{D}$ for the modulator.

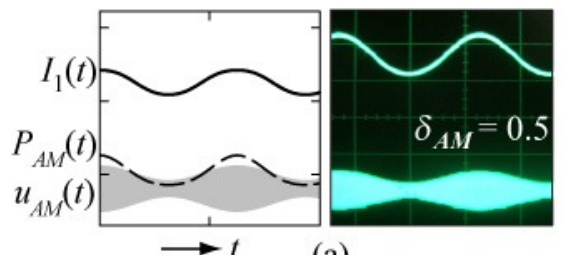

(a)

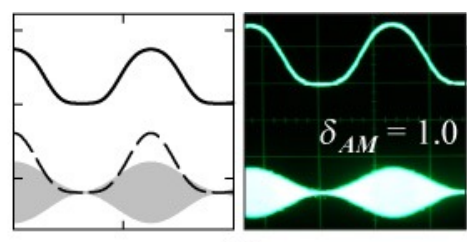

(b)

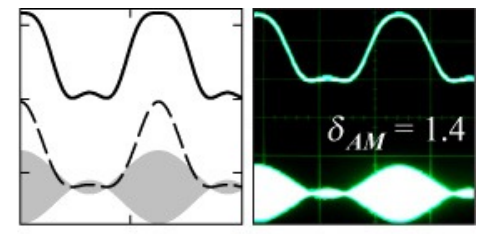

(c)

Figure 5. A series of calculated $u_{A M}(t)$ modulated electric signal amplitude and $I_{1}(t)$ diffracted intensity curves at different modulation depths $\delta_{A M}$ of 0.5 (a), 1.0 (b) and 1.4 (c) with the corresponding measured oscillograms. The modulated acoustic power $P_{A M}(t) \sim u_{A M}^{2}(t)$ is also given for direct comparison to $I_{1}(t) \sim P_{A M}(t)$.

The AM modulated input electric signal can be described as

$$
u_{i n}(t)=u_{A M}(t) \cos \left(2 \pi f_{a} t\right)=U_{0}\left[1+\delta_{A M} \cos \left(2 \pi f_{A M} t\right)\right] \cos \left(2 \pi f_{a} t\right)
$$

with modulation amplitude $u_{A M}(t)$, modulation frequency $f_{A M}$ and the parameter $\delta_{A M}$ defined as the modulation depth. The significance of the modulation depth can be readily demonstrated by setting up the internal AM modulation with $f_{A M}=400 \mathrm{~Hz}$. Figure 5 depicts a series of calculated electric $u_{A M}(t)$ to diffracted intensity $I_{1}(t)$ curves at different $\delta_{A M}$ values with the corresponding measured oscillograms. The modulated acoustic power, proportional to the squared modulated amplitude $P_{A M}(t) \sim u_{A M}^{2}(t)$, is also plotted in Figure 5. From these measurements, the low distortion region at $\delta_{A M}<1$ and the high distortion region at $\delta_{A M}>1$ with the apparent foldback around the nodes can be identified. In Figure 6a, we can demonstrate the combined effect of large $\delta_{A M}$ and that of high acoustic power at which 
the foldback appears at the signal peaks due to the nonlinear transfer function of Equation (13). The setup can be used even to visualize the temperature dependence of the transfer function: the peak foldback in the modulated intensity of the diffracted beam (Figure 6b) will increase as the average acoustic power inside the cell is reduced (Figure 6c) due to the shift of the transfer function towards higher driving powers. The average power reduction can be accomplished by turning on the switching modulator of the combined AO driver. The modulator periodically switches on and off the input of the power amplifier at a duty cycle of 50\% hence providing the same peak power at halved average power.

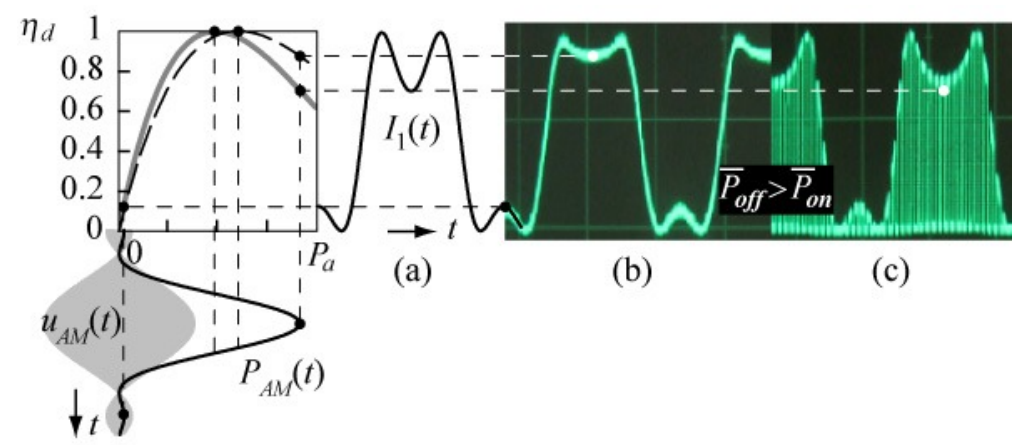

Figure 6. Demonstration of the combined effect of over-modulation at $\delta_{A M}=1.4$ yielding a foldback at signal nodes and that of large acoustic power $P_{a}$ at which the foldback appears at signal peaks due to the nonlinear modulation transfer function (a). Temperature dependent shift of the transfer function (from solid gray to dashed curve) is visualized in oscillograms (b) and (c): the average acoustic power $\bar{P}_{\text {off }}$ inside the cell in (b) is reduced to $\bar{P}_{o n}$ in (c) by turning on the switching modulator with switching frequency of $10 \mathrm{kHz}$ and duty cycle of $50 \%$.

Finally, to complete the AO modulation basics, some useful bandwidth must be added to the modulation. Recalling Figure 1c, we know that an incident optical plane wave $\mathbf{k}_{0}$ interacting with an acoustic plane wave $\mathbf{k}_{a}$ produces an optical plane wave $\mathbf{k}_{1}$ at Bragg condition. When the amplitude of an acoustic wave is AM modulated with a signal of bandwidth $\Delta f_{A M}$, it has frequency components within a band $f_{A M, 0} \pm \Delta f_{A M}$ that must interact with the incident optical wave. This can be done if the divergence $\delta \theta_{0} \approx \lambda_{0} / D$ of the incident optical beam of width $D$ is matched to the acoustic divergence $\delta \theta_{a}$ given in Equation (8) so that the $\Delta S\left(\Delta \theta_{a}\right)$ components of the angular spectrum of sound within $\Delta \theta_{a}=\delta \theta_{a}$ will diffract the components of the light beam within $\Delta \theta_{0}=\delta \theta_{0}$ similarly to that in Figures 2a-b:

$$
\Delta \theta_{a}=\delta \theta_{a}=\delta \theta_{0} \approx \frac{\lambda_{0}}{D}, \quad \Delta \theta_{a}=\Delta \theta_{0} \approx \lambda_{0} \Delta \frac{1}{\lambda_{a}}=\frac{\lambda_{0}}{v_{a}} \Delta f_{A M}
$$

From Equation (15), the $\Delta f_{A M}$ bandwidth of the modulator can be expressed as

$$
\Delta f_{A M}=\frac{1}{\tau_{D}}, \quad \tau_{D}=\frac{D}{v_{a}},
$$

where the transit time $\tau_{D}$ of sound across the light beam is introduced. Definition of $\tau_{D}$ holds for the collimated beam of the deflector in Figure 4a and the focused beam of the modulator in Figure 4b where the beam waist diameter is taken. The transit time can easily be measured by turning on the switching modulator of the setup and measuring the rise time of the square wave modulation of the output intensity with and without the lens pairs.

Now everything has been set to assemble the AO setup as a free space sound transmitter and demodulation system according to Figure 7. The most stunning experience is playing music fed from an input source to the AO cell via the external AM input of the RF generator. The free space path is identified as the 1st order beam of the AO cell fed to the photodetector. Any device that acts as an active speaker can be used for directly demodulating the output signal of the photodetector. Now students are free to play around with any parameters of the modulator/demodulator stages. 


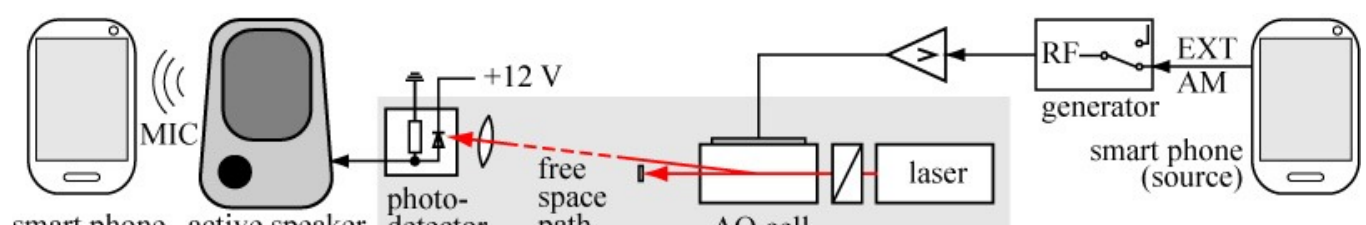

Figure 7. Block scheme of a free space sound transmitter and demodulation system built upon the basic AO setup.

\section{MSC LEVEL ACOUSTO-OPTICS IN APPLIED PHYSICS LABORATORY}

Within the framework of the MSc in Physics, applied acousto-optics is trained in the Applied Physics Laboratory of the Applied Physics specialization. The laboratory experiment focuses on the investigation of an AO tunable filter with mono- and polychromatic light sources to learn about spectral analysis and synthesis.

A major feature of an AO filter is that its narrow spectral transmission can be electronically tuned over a wide spectral range. There are some considerations to successfully realize an AO filter of Figure 8b. Whereas the devices studied earlier typically operate at a single wavelength of a laser source, an AO filter must operate in a wide spectral tuning range $\Delta \lambda=\lambda_{\max }-\lambda_{\min }$ utilizing a light source with broad and, ideally, uniform emission in this range. The period of the $\mathrm{AO}$ gratings constant, equal to the acoustic wavelength, can be tuned by the acoustic frequency to change the optical wavelength at which the Bragg condition is satisfied in a preferably narrow spectral bandwidth $\delta \lambda$. As such, the AO filter is an inverse deflector: at each wavelength a narrow diffraction bandwidth, hence a high resolution $\Re=\Delta \lambda / \delta \lambda$, is realized even at large input beam divergence as well as the change of the position of the diffracted beam is avoided.

(a)

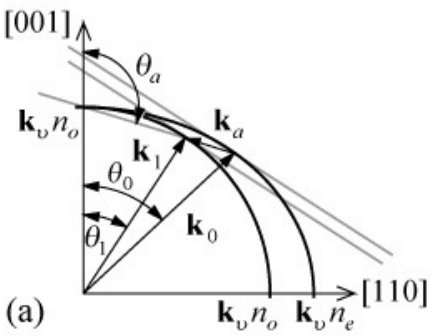

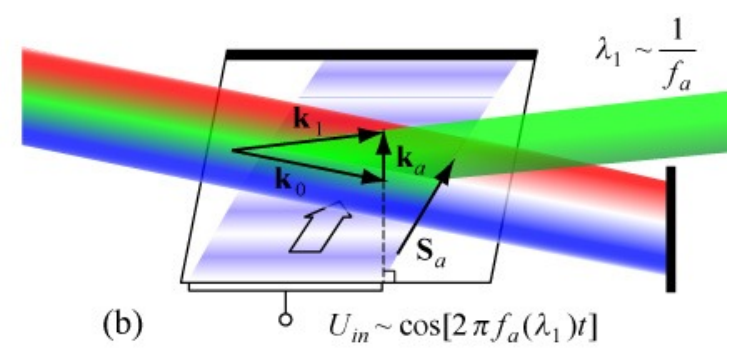

(b)

(b) for the AO filter with characteristic input and output Figure 8. Anisotropic AO interaction (a) and typical geometry (b) for the AO filter witt
signals. Note the different propagation direction of acoustic energy $\mathbf{S}_{a}$ and wavefront $\mathbf{k}_{a}$.

To satisfy all these conditions simultaneously, anisotropic AO interaction must be employed. In an anisotropic interaction, the polarization of the diffracted beam is changed. As a result, due to the birefringence of the medium, the refractive index for the diffracted beam also changes so the angles of incidence and diffraction will no longer be symmetric. Equation (6) for wave vector matching also hold for $m=1$, but now the incident and diffracted wave vectors have different lengths, as illustrated in Figure 8a. A wavelength outside the Bragg condition will introduce a wave vector mismatch with magnitude $\Delta k$ so the normalized transmittance $T_{\lambda}(\Delta k)$ will drop as

$$
T_{\lambda}(\Delta k)=\operatorname{sinc}^{2}\left(\frac{\Delta k L}{2}\right) .
$$

To compensate for the larger divergence of a broadband source, the Bragg condition must not change upon the infinitesimal change of the incident angles in the interaction plane $\delta(\Delta k) / \delta \theta_{0}=0$ or the orthogonal plane $\delta(\Delta k) / \delta \phi_{0}=0$ imposing the condition for parallel planes ${ }^{16}$ of Figure 8a.

The breadboard of the experimental setup comprises a white LED source (Cree Xlamp XPCWHT-L1) with $150 \mu \mathrm{m}$ pinhole and a collimator lens, an in-house made $\mathrm{TeO}_{2}$ based $\mathrm{AO}$ filter on kinematic mount and a photodetector unit 
connected to an oscilloscope. The AO filter is driven by a programmable RF generator (Rohde \& Schwarz SMY01). Figure 9 plots some of the parameters that can be determined during the experiment. The wavelength dependent curves can be taken by using a fiber optic spectrometer. Alternatively, the tuning curve $f_{a}\left(\lambda_{v}\right)$ can be extrapolated from data taken at 3 known (laser) wavelengths, then the parameters can be taken versus $f_{a}$ and transformed against $\lambda_{v}$.
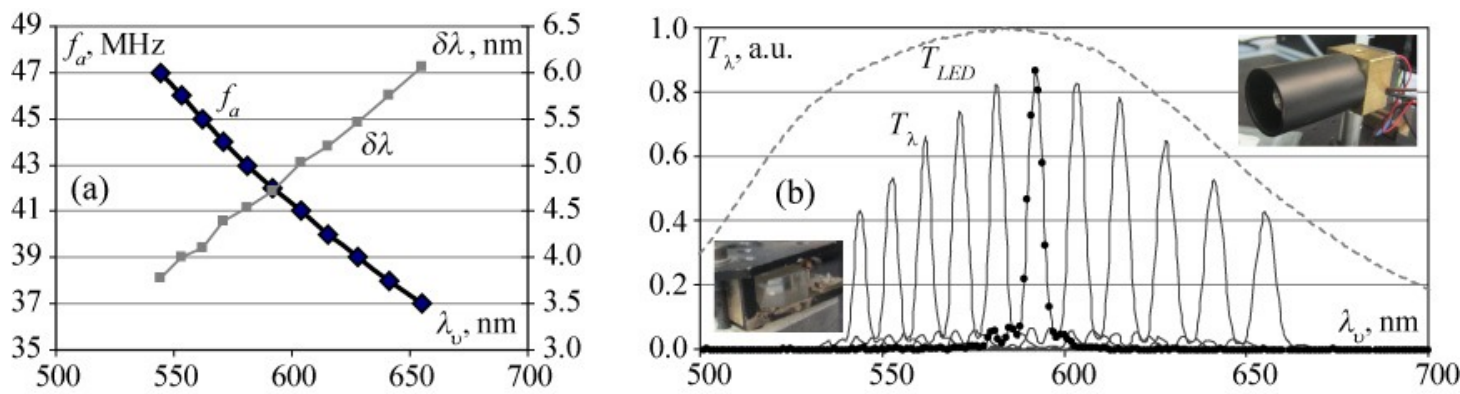

Figure 9. Typical AO filter parameters determined during the experiment: (a) tuning curve $f_{a}\left(\lambda_{v}\right)$ and spectral bandwidth $\delta \lambda\left(\lambda_{v}\right)$; (b) filter transmission curves $T_{\lambda}\left(\lambda_{v}, \Delta k\right)$ and LED spectral emission $T_{L E D}\left(\lambda_{v}\right)$. Insets: AO filter (bottom), LED (top).

\section{E-LEARNING ELEMENTS IN ACOUSTO-OPTICS}

Recently, elements of e-Learning has been defined or introduced in the training of acousto-optics to help students' laboratory preparation, work and reporting. A Moodle based learning management site (LMS) on a local network attached storage (NAS) server was established with a collection of course contents in downloadable PDF format or, recently, with online SCORM content. Although course materials are available from the student's site at the department homepage, LMS offers a stricter way of management of laboratory courses.

First, preparation can be checked by scoring that students have read the required material in time prior to the laboratory. Also, a 'pre-lab' test can be set up outside the laboratory contact hours saving extra time for the exercise. The online content can be used throughout the exercise and it is available on mobile phones with animation, occasionally (Figure 10a). Mobile devices offer m-Learning ${ }^{17}$, so that they can form an integral part of the experiment, in addition to content viewing. As an example, in the advanced training of the basic AO setup, a mobile device can generate signal inputs for the free space transmission of Figure 7 through its headset output and record the sound of the speaker. In both setups, students are encouraged to take photos of the screen, for qualitative or even quantitative evaluation in their report. Finally, post-lab tests (Figure 10b) and online submission of reports can be initiated helping tutors in students' evaluation such as automated checking of report submission. Whereas pre-lab tests aim at scoring students' (theoretical) knowledge in preparation for the laboratory, post-lab tests are more practical to measure those skills gained during the experiments, as in Figure 10b that shows a fairly 'visual’ question.
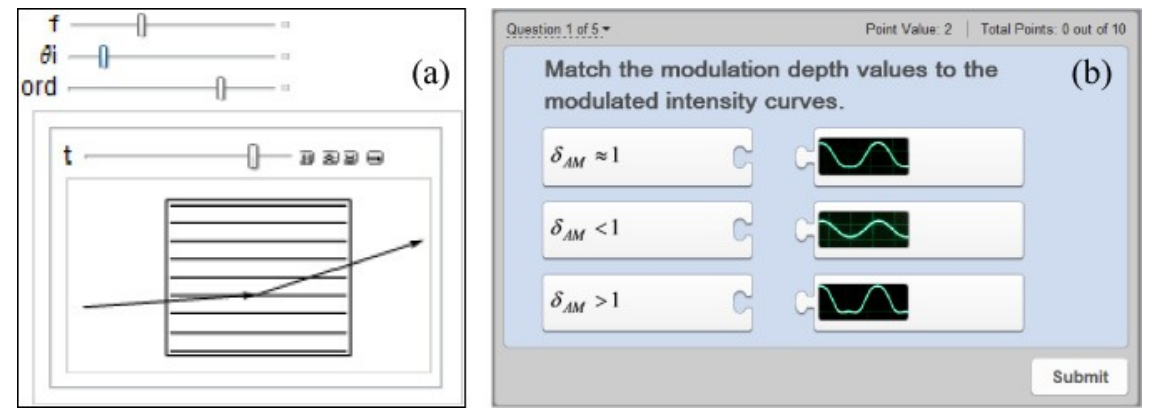

Figure 10. Example of online content for students attending AO laboratory: AO effect animation (a) and post-lab test (b). 


\section{CONCLUSIONS}

In this paper, an overview of training of acousto-optics has been given with a step-by-step introduction of more basic setup used at BSc and advanced levels, and with a brief description of an MSc level experiment. We conclude that both laboratories directly offer the fundamental knowledge for both undergraduate courses and advanced research in utilizing the AO effect. The training can be beneficial in a PhD thesis work ${ }^{13}$ or in a photonics related profession. For example, the concept of modulation can be readily extended to standing acoustic wave AO cells with self modulation to get mode locking of lasers with large modulation depth and low duty factor ${ }^{4}$. Deflector basics can be extended to multidimensional scanners such as those used in 3D beam steering in two-photon microscopes ${ }^{11}$. Filter configurations can be used for pulse shaping of femtosecond lasers ${ }^{12}$. Finally, e-learning elements have been introduced that help improve students' laboratory experience and tutors' evaluation efforts. As e-learning is quite new in this context, conclusions are awaiting.

\section{ACKNOWLEDGEMENTS}

This work has been partially funded by "TÁMOP-4.1.1.C-12/1/KONV-2012-0005 - Preparation of the concerned sectors for educational and R\&D activities related to the Hungarian ELI project” supported by the European Union and co-financed by the European Social Fund.

\section{REFERENCES}

[1] Brillouin, L., "Diffusion of Light and X-rays by a Transparent Homogeneous Body: Influence of thermal agitation," Annales de Physique 17, 88-122 (1922).

[2] Debye, P. and Sears, F. W., "On the scattering of light by supersonic waves," PNAS 18(6), 409-414 (1932).

[3] Lucas, R. and Biquard, P., "Optical properties of solid and liquid medias subjected to high-frequency elastic vibrations," Journal de Physique 71, 464-477 (1932).

[4] Eichler, H. J., Barocsi, A., Jakab, L. and Liu, B., "Acousto-optic mode-locker for Nd:lasers using paratellurite," Appl. Phys. B - Photo. 53(3), 194-197 (1991).

[5] Maak, P., Jakab, L., Richter, P., Eichler, H. J., and Liu, B., "Efficient acousto-optic Q-switching of Er:YSGG lasers at $2.79 \mu \mathrm{m}$ wavelength," Appl. Optics 39(18), 3053-3059 (2000).

[6] Maák, P., Jakab, L., Barócsi, A., and Richter, P., "Recent Developments and Results in 2D Acousto-Optic Light Deflection," Proc. SPIE 3388, 48-53 (1998).

[7] Maak, P., Lenk, S., Jakab, L., Barocsi, A. and Richter, P., "Optimization of transducer configuration for bulk acousto-optic tunable filters," Opt. Commun. 241(1-3), 87-98 (2004).

[8] Barocsi, A., Jakab, L. and Richter, P., "Efficient, extremely low frequency acousto-optic shifter for optical heterodyning applications," Proc. SPIE 2240, 108-113 (1994).

[9] Barocsi, A., Jakab, L., Szarvas, G., Richter, P. and Szonyi, I., "Recent developments and results in acoustooptic signal processing," Proc. SPIE 2754, 21-30 (1996).

[10] Jakab, L., Barocsi, A., Richter, P., Szonyi, I. and Rath, T., "Test measurement and calibration problems of a direction finding system based on a five channel DOA processor," Proc. SPIE 2240, $43-49$ (1994).

[11] Katona, G., Szalay, G., Maak, P., Kaszas, A., Veress, M., Hillier, D., Chiovini, B., Vizi, E. S., Roska, B. and Rozsa, B., "Fast two-photon in vivo imaging with three-dimensional random-access scanning in large tissue volumes," Nature Methods 9(2), 201-208 (2012).

[12] Maák, P., Kurdi, G., Barócsi, A., Osvay, K., Kovács, A. P., Jakab, L. and Richter, P., "Shaping of ultrashort laser pulses using bulk acousto-optic filter," Appl. Phys. B - Lasers O. 82(2), 283-287 (2006).

[13] Mihajlik, G., Barócsi, A. and Maák, P., "Measurement and general modeling of optical rotation in anisotropic crystal,” Opt. Commun. 310, 31-34 (2014).

[14] Uchida, N. and Niizeki, N., "Acoustooptic deflection materials and techniques," Proc. IEEE 61(8), 1073-1092 (1973).

[15] Saleh, B. E. A. and Teich, M. C., [Fundamentals of Photonics 2nd Edition], John Wiley \& Sons, Inc., Hoboken, New Jersey, 804-833 (2007).

[16] Chang, I. C., "Analysis of the noncollinear acousto-optic filter," Electron. Lett. 11(25/26), 617-618 (1975)

[17] Calvo, M. L., "m-Learning and holography: Compatible techniques?," Proc. SPIE 9289, 928903 (2014) 